ARTICLE OPEN

Check for updates

\title{
Band gap crossover and insulator-metal transition in the compressed layered $\mathrm{CrPS}_{4}$
}

Resta A. Susilo $\mathbb{D}^{1 凶}$, Bo Gyu Jang ${ }^{1,2}$, Jiajia Feng ${ }^{1}$, Qianheng Du $\mathbb{D i D}^{3,4}$, Zhipeng Yan ${ }^{1}$, Hongliang Dong ${ }^{1}$, Mingzhi Yuan ${ }^{1}$, Cedomir Petrovic ${ }^{3,4}$, Ji Hoon Shim ${ }^{2}$, Duck Young Kim ${ }^{1}$ and Bin Chen ${ }^{1 凶}$

Two-dimensional van der Waals (vdW) magnetic materials have emerged as possible candidates for future ultrathin spintronic devices, and finding a way to tune their physical properties is desirable for wider applications. Owing to the sensitivity and tunability of the physical properties to the variation of interatomic separations, this class of materials is attractive to explore under pressure. Here, we present the observation of direct to indirect band gap crossover and an insulator-metal transition in the vdW antiferromagnetic insulator $\mathrm{CrPS}_{4}$ under pressure through in-situ photoluminescence, optical absorption, and resistivity measurements. Raman spectroscopy experiments revealed no changes in the spectral feature during the band gap crossover whereas the insulator-metal transition is possibly driven by the formation of the high-pressure crystal structure. Theoretical calculations suggest that the band gap crossover is driven by the shrinkage and rearrangement of the $\mathrm{CrS}_{6}$ octahedra under pressure. Such high tunability under pressure demonstrates an interesting interplay between structural, optical and magnetic degrees of freedom in $\mathrm{CrPS}_{4}$, and provides further opportunity for the development of devices based on tunable properties of 2D vdW magnetic materials.

npj Quantum Materials (2020)5:58; https://doi.org/10.1038/s41535-020-00261-x

\section{INTRODUCTION}

Ever since the successful exfoliation of stable single-layer graphene ${ }^{1}$, research on layered two-dimensional van der Waals (2D vdW) materials has attracted significant interests in the past decade. One of the current focuses is the investigation of magnetic 2D vdW materials, which is quite attractive from both a fundamental physics and practical application perspective (e.g., refs $\left.{ }^{2-4}\right)$. In principle, the discovery of atomically thin materials with intrinsic ferromagnetism and high-magnetic ordering temperature could open up opportunities to realize spintronic devices, such as molecular quantum devices and high-density ultrathin storage devices. Indeed, the recent groundbreaking experimental observations of the intrinsic magnetism in atomically thin $\mathrm{FePS}_{3}$, $\mathrm{Crl}_{3}, \mathrm{Cr}_{2} \mathrm{Ge}_{2} \mathrm{Te}_{6}$, and $\mathrm{Fe}_{3} \mathrm{GeTe}_{2}{ }^{5-11}$ have pushed this class of materials to be promising candidates for possible applications in spintronic technology, as they can serve as building blocks for magnetic vdW heterostructures.

In order to explore the wider applications of 2D vdW materials, it is necessary to tune their optical, electronic, and magnetic properties. Several attempts have been made to tune the properties of $2 \mathrm{D}$ vdW magnets, such as by intercalation, gating, electrostatic doping, and changing layer thickness to name a few (e.g., refs ${ }^{9,12-17}$ ). Pressure or compressive strain might also be an alternative way to tune the properties of the $2 \mathrm{D} v \mathrm{vdW}$ magnets without introducing disorder or impurities to the system. It has been recently shown that pressure can be effectively used to modify the magnetic, electronic, and optical properties of 2D vdW magnets both in bulk and atomically thin samples ${ }^{18-29}$.

Among the 2D vdW magnetic materials, $\mathrm{CrPS}_{4}$ is currently enjoying a surge of interest ${ }^{3-35} \cdot \mathrm{CrPS}_{4}$ was first synthesized in the 1970 s and found to possess a monoclinic symmetry $(C 2 / \mathrm{m}$ space group $)^{36}$. In the structure, puckered layers of $S$ atoms are arranged in hexagonal close packing parallel to the a-axis, whereas the $\mathrm{Cr}$ atoms form a square lattice. Each $\mathrm{Cr}$ atom is surrounded by six $\mathrm{S}$ atoms forming a slightly distorted octahedron, and three $\mathrm{CrS}_{6}$ octahedra are connected by a single P atom (Fig. 1(a)). It is an antiferromagnetic semiconductor with a band gap of $\sim 1.4 \mathrm{eV}^{30,37}$. Previous magnetic studies suggested a C-type antiferromagnetic structure (antiferromagnetic interaction within a layer and ferromagnetic interaction between the layer) below the Néel temperature $\left(T_{N}\right)$ of $36 \mathrm{~K}^{38}$. However, recent neutron diffraction and torque magnetometry investigations revealed an A-type AFM structure with the intralayer ferromagnetic interaction and interlayer antiferromagnetic coupling along the $c$ axis ${ }^{35}$. X-ray spectroscopy studies demonstrated that the $\mathrm{Cr} 3 d$ bands are localized within the energy gap in the vicinity of the Fermi level, where the lower and higher energy of the band structure is dominated by the non-bonding $S 3 p$ band and the $S 3 s$ band, respectively ${ }^{39}$. Theoretical calculations predicted that $\mathrm{CrPS}_{4}$ is stable in the monolayer form while retaining the intrinsic magnetism and semiconducting properties ${ }^{40}$. Lee et al. ${ }^{30}$ reported thickness-dependence studies of the structural, vibrational, and optical properties of $\mathrm{CrPS}_{4}$ where they observed the disappearance of Raman peaks and weakening of the photoluminescence signal in the monolayer limit due to photodegradation of the thin sample ${ }^{30}$. Symmetry breaking was found to induce the appearance of Fano resonance in the photoluminescence spectra of $\mathrm{CrPS}_{4}$ collected below the antiferromagnetic transition ${ }^{34}$.

With its rich optical and electronic properties, exploring the tunability of $\mathrm{CrPS}_{4}$ by the application of pressure is beneficial, not only to improve the clear understanding of the physical properties but also to investigate the wider potential applications of this material. However, the high-pressure behavior of $\mathrm{CrPS}_{4}$ remains unexplored to date. By comparison, high-pressure investigations

\footnotetext{
${ }^{1}$ Center for High Pressure Science and Technology Advanced Research, 201203 Pudong, Shanghai, China. ${ }^{2}$ Department of Chemistry, Pohang University of Science and Technology, Pohang 37673, Korea. ${ }^{3}$ Condensed Matter Physics and Materials Science Department, Brookhaven National Laboratory, Upton, NY 11973 , USA. ${ }^{4}$ Department of

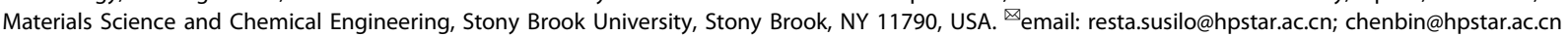



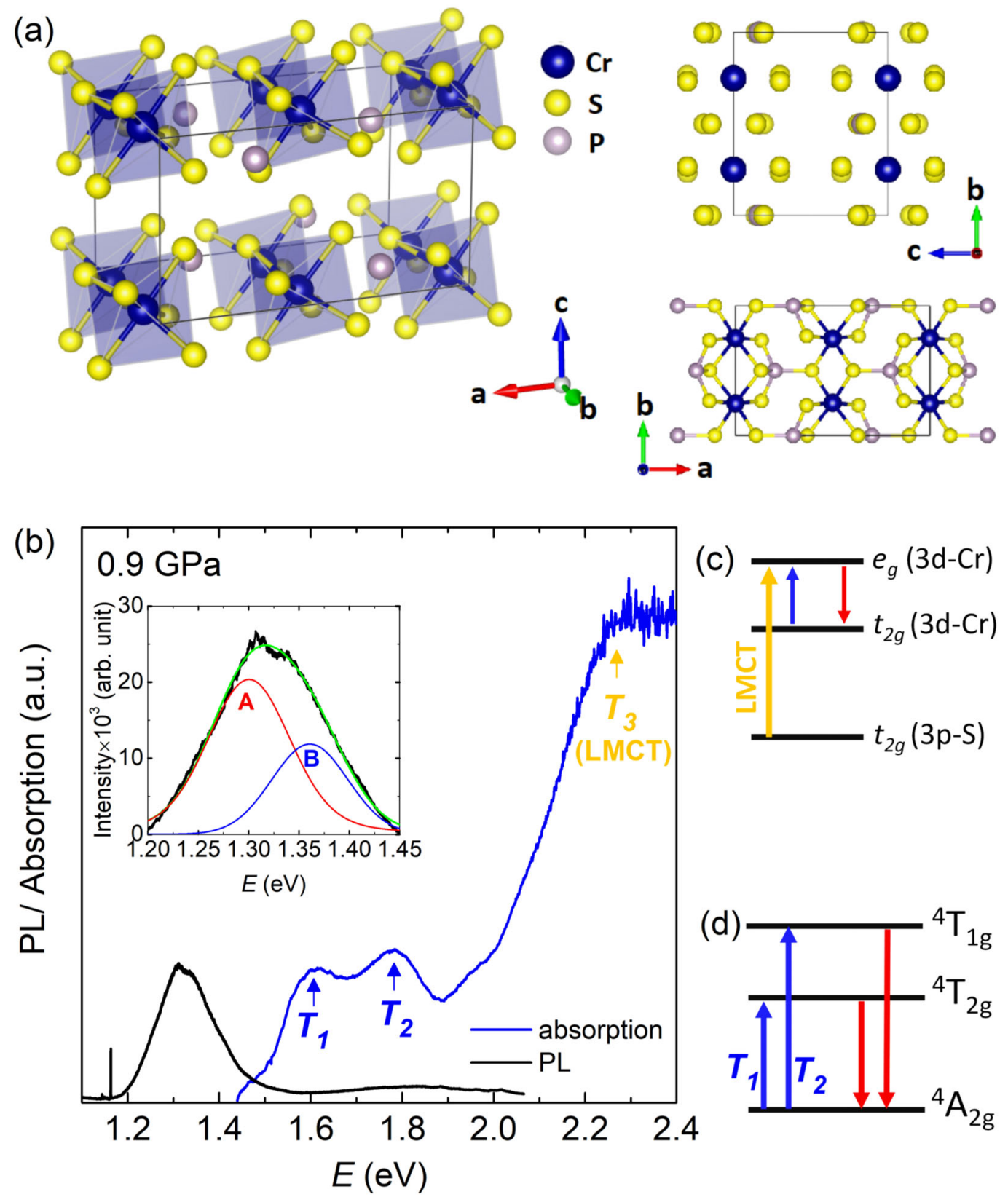

(c)
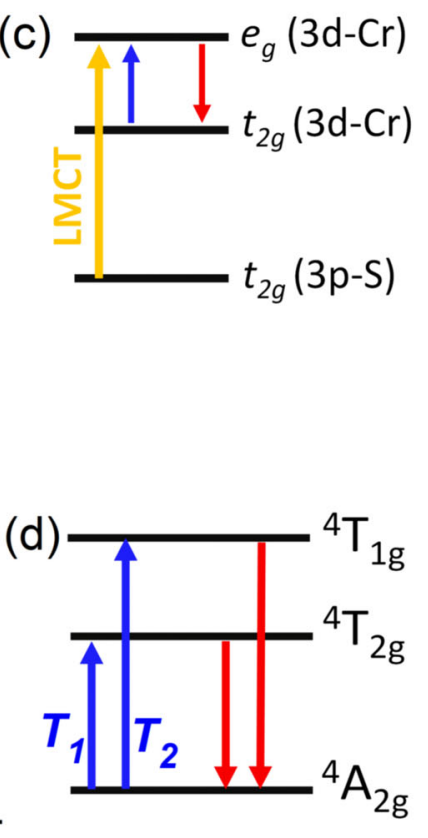

Fig. 1 Crystal structure and optical characterization of $\mathrm{CrPS}_{\mathbf{4}}$. a Crystal structure of $\mathrm{CrPS}_{4}$ with the $\mathrm{C2} / \mathrm{m}$ space group. $\mathbf{b}$ Room temperature photoluminescence and optical absorption spectra of $\mathrm{CrPS}_{4}$ below $1 \mathrm{GPa}$. Inset shows the deconvoluted PL spectrum fitted with two Voightian peaks. c Molecular orbital energy diagram involved in the optical transitions of $\mathrm{CrPS}_{4}$. The blue and red arrows indicate $d-d$ transitions and the orange arrow represents a possible ligand-to-metal charge-transfer (LMCT) transition. $\mathbf{d}$ Configurational diagram of $\mathrm{Cr}^{3+}$ for the observed absorption (blue arrows) and PL (red arrows) spectra.

on the closely related systems such as $\mathrm{TMP}(\mathrm{S}, \mathrm{Se})_{3}(\mathrm{TM}=$ transition metal) in which the TM ions form a layered honeycomb lattice, have revealed Mott insulator-metal and structural transitions in $\mathrm{MnPS}_{3}{ }^{18}, \mathrm{FePS}_{3}{ }^{20,25,27}, \mathrm{FePSe}_{3}{ }^{25}$, and recently Mott isostructural transition in $\mathrm{V}_{0.9} \mathrm{PS}_{3}{ }^{26}$.

In this work, we examine the effects of pressure on the optical, electronic, and vibrational properties of layered $\mathrm{CrPS}_{4}$ through the use of optical absorption, photoluminescence, resistivity, and Raman spectroscopy measurements. We observed anomalous changes in the photoluminescence and optical absorption spectra on compression above $\sim 3 \mathrm{GPa}$. Further compression leads to an insulator-metal transition, which begins above $15 \mathrm{GPa}$ as evidenced by the optical absorption and resistivity data. Raman spectroscopy measurements indicates that the crystal structure is stable below $10 \mathrm{GPa}$. However, the Raman spectra significantly changes above $16 \mathrm{GPa}$, which suggests the formation of a highpressure phase accompanying the insulator-metal transition in $\mathrm{CrPS}_{4}$. Theoretical calculations show that the anomalies observed in the photoluminescence and optical absorption data above $3 \mathrm{GPa}$ are related to the direct to indirect band gap crossover, which is mainly driven by the rearrangement of the $\mathrm{CrS}_{6}$ octahedra under pressure.

\section{RESULTS AND DISCUSSION}

Optical properties under pressure

The photoluminescence $(\mathrm{PL})$ and optical absorption spectra of $\mathrm{CrPS}_{4}$ at the lowest pressure of our measurements $(P<1 \mathrm{GPa})$ are 

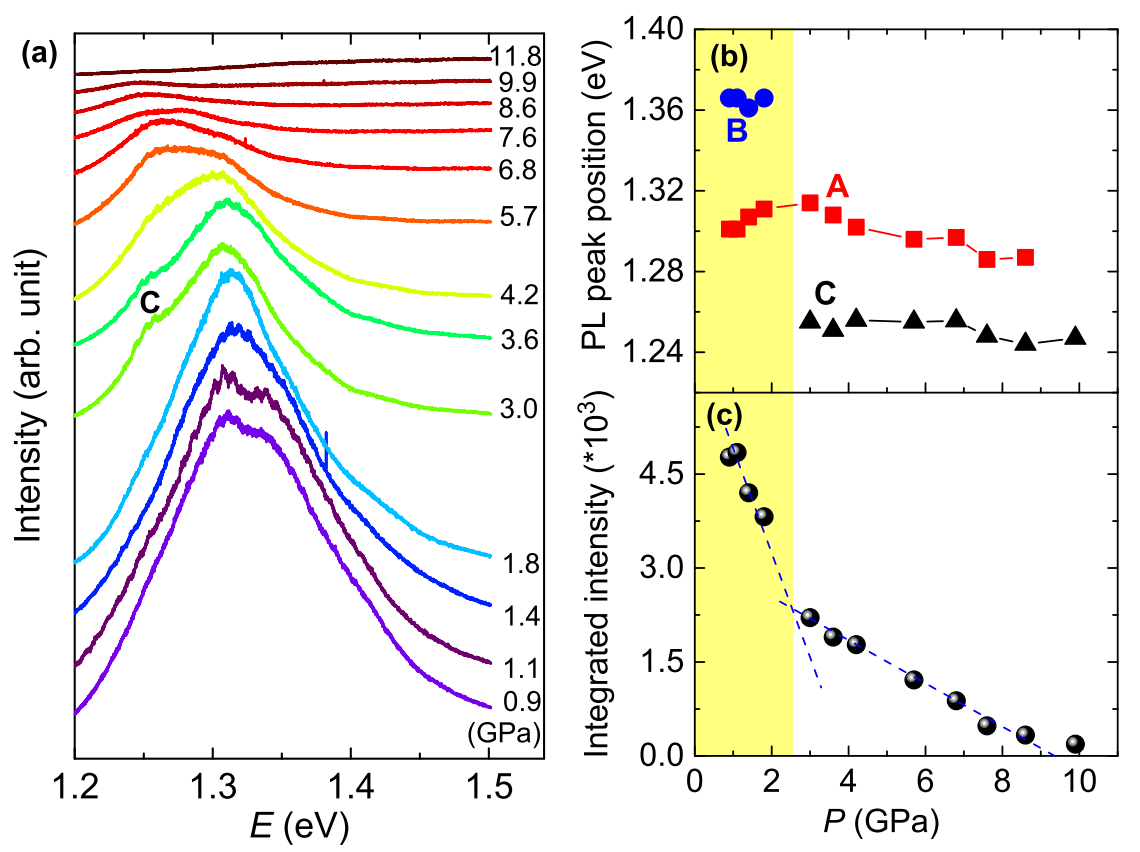

Fig. 2 High-pressure photoluminescence properties of $\mathrm{CrPS}_{4}$. a Room temperature PL spectra of $\mathrm{CrPS}_{4}$ as a function of pressure. The spectra are shifted for clarity. The derived pressure dependences of (b) PL peak position and (c) intensity of the PL peak integrated from 1.2 to $1.5 \mathrm{eV}$.

presented in Fig. 1(b). Both spectra are similar to those reported previously at ambient pressure, which suggests that the optical and electronic properties of the sample are not affected by the application of pressure $<1 \mathrm{GPa}$. As shown in Fig. 1(b), the sample at $0.9 \mathrm{GPa}$ exhibits a broad $\mathrm{PL}$ peak centered at $\sim 1.3 \mathrm{eV}$, in agreement with the PL spectrum at ambient conditions ${ }^{30,34}$. The PL spectrum consists of two Voightian components centered at at $\sim 1.3$ and $\sim 1.37 \mathrm{eV}$, respectively ( $\mathrm{A}$ and $\mathrm{B}$ components in the inset of Fig. 1(b)). Previous studies showed that the intensity of the PL peak of $\mathrm{CrPS}_{4}$ increases linearly with increasing laser power without any signs of saturation, ruling out the possible excitonic emission of the PL in $\mathrm{CrPS}_{4}{ }^{34}$. In $\mathrm{CrPS}_{4}$ crystal, the $\mathrm{Cr}^{3+}$ ion is located in a distorted octahedral environment formed by six $\mathrm{S}$ atoms. In this case, the octahedral crystal field will split the $3 d$ orbital of $\mathrm{Cr}^{3+}$ into $t_{2 g}$ and $e_{g}$ orbitals with a splitting energy of $\Delta \sim 2.0 \mathrm{eV}^{39}$ (Fig. 1(c)), and the electronic configuration of $\mathrm{Cr}^{3+}$ ( $d^{3}$ configuration, ${ }^{4} \mathrm{~F}$ term) splits into the ${ }^{4} \mathrm{~A}_{2 g}$ ground state and ${ }^{4} \mathrm{~T}_{2 g}$ and ${ }^{4} \mathrm{~T}_{1 g}$ excited states (Fig. $1(\mathrm{~d})$ ). The PL peak observed in $\mathrm{CrPS}_{4}$ has been previously attributed to the spin-allowed $d-d$ transition of the $\mathrm{Cr}^{3+}$ ion $^{30,34}$ (red arrows in Fig. 1(c, d)), which are often located around $1.6-2.2 \mathrm{eV}$ in the $\mathrm{Cr}$-based compounds ${ }^{41,42}$.

The optical absorption spectrum exhibits three clear features (marked by $T_{1}, T_{2}$, and $T_{3}$ ). The feature at $T_{3}$ has been assigned to the ligand-to-metal charge-transfer (LMCT) transition from the $3 p$ of the $\mathrm{S}$ band to the unoccupied $3 d$ band of the $\mathrm{Cr}$ atom ${ }^{30,39}$ (orange arrow in Fig. 1(c)). In the lower energy region, two weaker $\left(T_{1}\right.$ and $T_{2}$ ) peaks located at $\sim 1.6$ and $\sim 1.8 \mathrm{eV}$ can be observed. These two absorption peaks can be assigned to the spin-allowed $d$ $-d$ transitions (blue arrows in Fig. $1(\mathrm{c}, \mathrm{d}))^{30,34}$, which are forbidden according to the Laporte parity selection rule. Despite being forbidden, these $d-d$ transitions can be weakly allowed due to local symmetry breaking ${ }^{43}$. A large Stokes shift between the PL peak and the $T_{1}$ peak in the absorption spectra of $\sim 300 \mathrm{meV}$ is due to the Franck-Condon principle and strong electron-lattice coupling ${ }^{44}$. Both PL and absorption data, therefore, indicate that $\mathrm{CrPS}_{4}$ possesses a band gap of $\sim 1.3 \mathrm{eV}$, which is governed by the $\mathrm{Cr} d-d$ transition.

In order to investigate the evolution of electronic structures under pressure, we measured in-situ PL and optical absorption data. Figure 2(a) shows the PL spectra of single crystal $\mathrm{CrPS}_{4}$ at room temperature collected up to $11.8 \mathrm{GPa}$. Under compression to $1.8 \mathrm{GPa}$, the PL A peak slightly blue-shifts to higher energy whereas the position of the $B$ peak remains constant (Fig. 2(b) and Supplementary Fig. 3). Above 2 GPa the A peak gradually moves to lower energy and the B peak disappears while a new PL peak simultaneously appears at lower energy (marked by C in Fig. 2(a) and Supplementary Fig. 3). The A peak is no longer observable above $8 \mathrm{GPa}$ while the $\mathrm{C}$ peak remains up to $10 \mathrm{GPa}$, above which the entire PL signal of $\mathrm{CrPS}_{4}$ disappears.

The pressure dependence of the PL peak intensity (integrated from 1.2 to $1.5 \mathrm{eV}$ ) is shown in Fig. 2(c). The PL peak intensity of $\mathrm{CrPS}_{4}$ continuously decreases with increasing pressure and the PL signal completely disappears above $10 \mathrm{GPa}$. A clear change in slope is observed at around $2.5 \mathrm{GPa}$, similar to the pressure where the new PL $(C)$ peak starts to emerge. One possible explanation for the observed change in the PL spectra and intensities of $\mathrm{CrPS}_{4}$ under pressure might be the band gap crossover (direct to indirect band gap). Similar behavior in the PL spectra has been observed previously in several systems such as $\ln \mathrm{P}^{45}, \ln \mathrm{Se}^{46}$ and monolayer $\mathrm{MoS}_{2}{ }^{47}$, and has been attributed to the direct-indirect gap crossover induced by pressure. However, given that the PL signal of $\mathrm{CrPS}_{4}$ arises from the parity forbidden $d-d$ transition, which is sensitive to the $\mathrm{CrS}_{6}$ octahedra, the anomalous behavior of PL data above $3 \mathrm{GPa}$ could be related to a subtle change in the crystal structure, possibly a modification of the $\mathrm{CrS}_{6}$ octahedra under pressure.

In Fig. 3(a), we present the Tauc plot $\left((a h v)^{2}\right.$ versus the photon energy $h v$ ) of the low-energy region of the room temperature optical absorption data measured up to $27 \mathrm{GPa}$. Given that the strong absorption edge at $\sim 2 \mathrm{eV}$ is related to the LMCT transition, we focus our analyses on the evolution of band gap associated with the $d-d\left(T_{1}\right.$ and $\left.T_{2}\right)$ transitions as described in Fig. 1 and Supplementary Fig. 4. The derived band gaps are shown in Fig. 3 (b). The band gaps for the $T_{1}$ and $T_{2}$ transitions at $0.8 \mathrm{GPa}$ are $\sim 1.5$ and $\sim 1.6 \mathrm{eV}$, respectively. These band gaps are relatively unchanged as the pressure is increased to $\sim 4 \mathrm{GPa}$. Interestingly, the $T_{1}$ peak and its associated band edge disappears above $4 \mathrm{GPa}$, whereas the $T_{2}$ peak remains (see Supplementary Fig. 5). The band gap for the $T_{2}$ transition slightly increases above $5 \mathrm{GPa}$ before it 

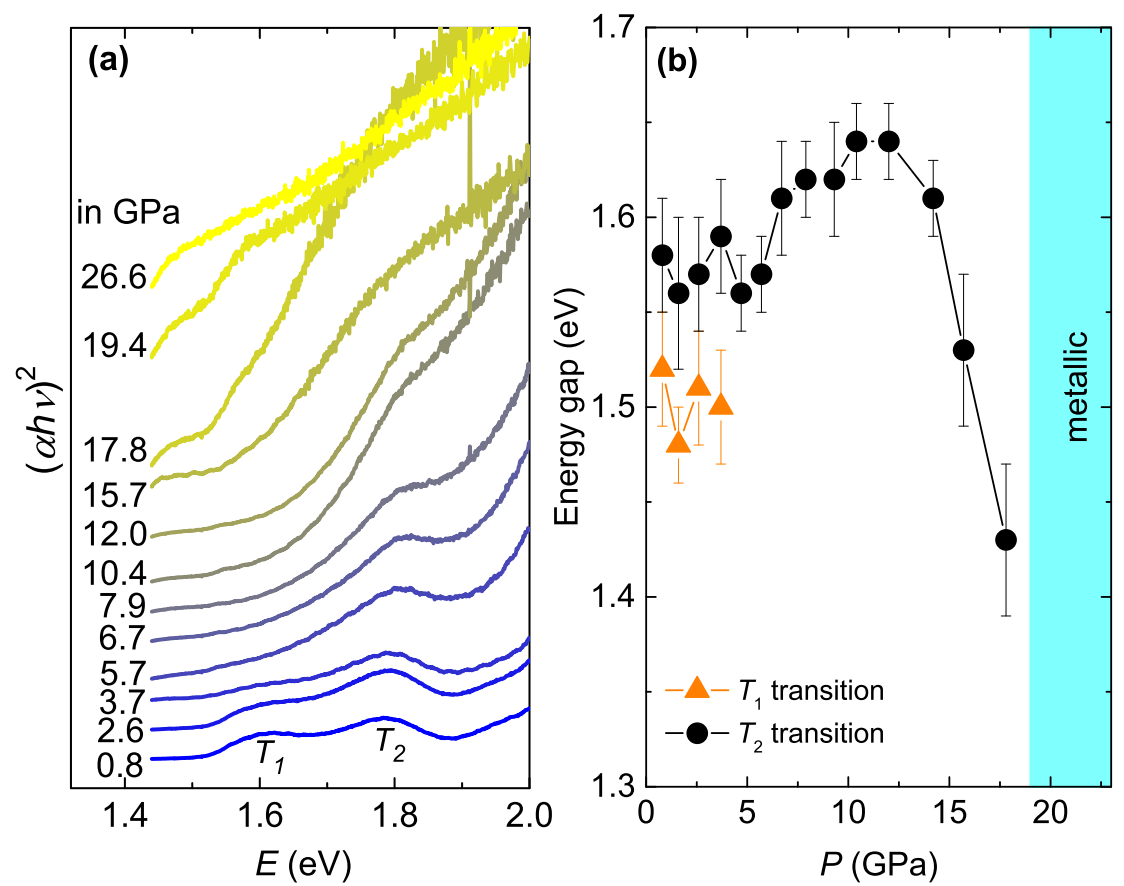

Fig. 3 High-pressure evolution of optical absorption of $\mathbf{C r P S}_{\mathbf{4}}$. a Tauc plot ((ahv) ${ }^{2}$ versus the photon energy hv) of room temperature optical absorption spectra of $\mathrm{CrPS}_{4}$ at various pressures. The spectra are shifted for clarity. $\mathbf{b}$ The derived optical band gaps associated with the $\mathrm{Cr} d-d$ $\left(T_{1}\right.$ and $\left.T_{2}\right)$ transitions in $\mathrm{CrPS}_{4}$ at high pressures.
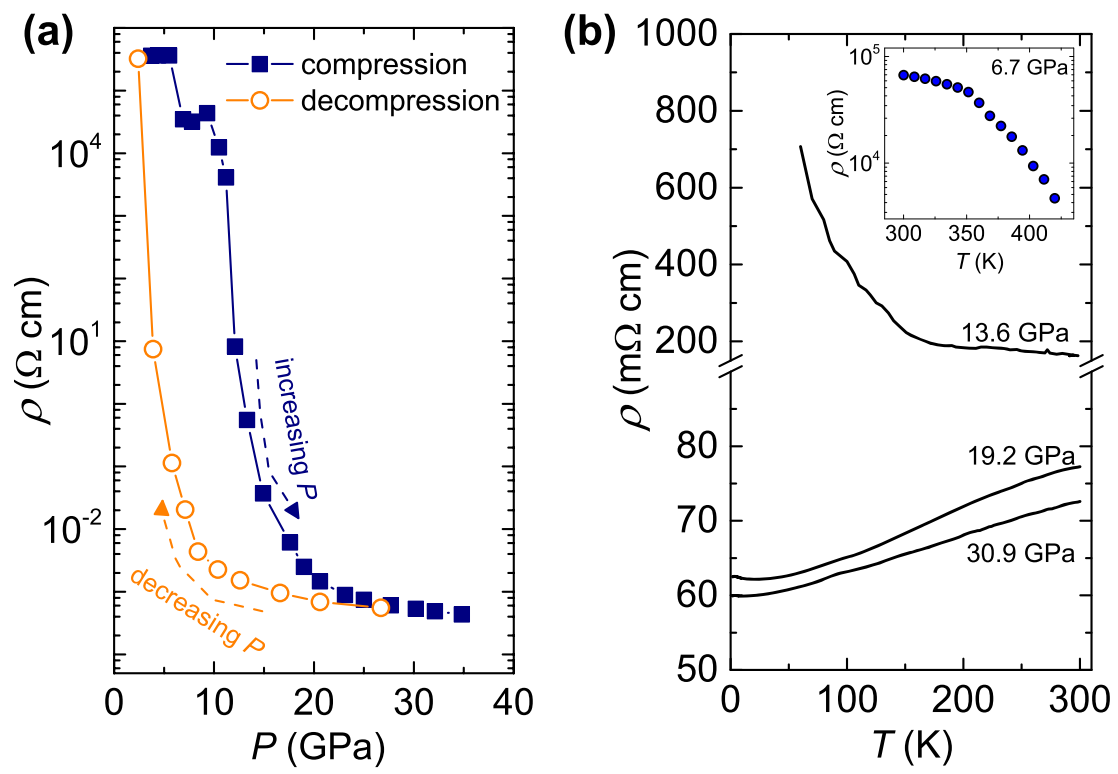

Fig. 4 Electrical transport properties of $\mathrm{CrPS}_{\mathbf{4}}$ under pressure. a Pressure-dependence resistivity of $\mathrm{CrPS}_{4}$ at room temperature. b Temperature-dependence resistivity of $\mathrm{CrPS}_{4}$ obtained at various pressures. Inset shows the temperature-dependence resistivity at $6.7 \mathrm{GPa}$ measured from room temperature to $420 \mathrm{~K}$.

starts to decrease above $12 \mathrm{GPa}$. A clear absorption edge is no longer observable above $19 \mathrm{GPa}$, which suggests a pressureinduced metallization in $\mathrm{CrPS}_{4}$ above $18 \mathrm{GPa}$. The overall behavior of the band gap derived from the absorption data (Fig. 3(b)) is similar to that of the PL data. In particular the band gap of $\mathrm{CrPS}_{4}$, governed by the $\mathrm{Cr} d-d$ transition, seems to undergo an anomalous change above $4 \mathrm{GPa}$. The band gap slightly increases on further compression to $\sim 12 \mathrm{GPa}$, above which it begins to narrow and finally closes above $19 \mathrm{GPa}$.
Electronic transport properties

The effects of pressure to the electronic properties and possible pressure-induced metallization of $\mathrm{CrPS}_{4}$ is further investigated by electrical transport measurements. In Fig. 4(a) we present the pressure dependence of resistivity of $\mathrm{CrPS}_{4}$ measured at ambient because the resistivity value of $\mathrm{CrPS}_{4}$ below $5 \mathrm{GPa}$ was beyond the sensitivity of the equipment used. The resistivity values between 5 and $10 \mathrm{GPa}$ are in the order of $\sim 10^{5} \Omega \mathrm{cm}$, which indicates an extremely insulating sample. However, on increasing pressure, the 

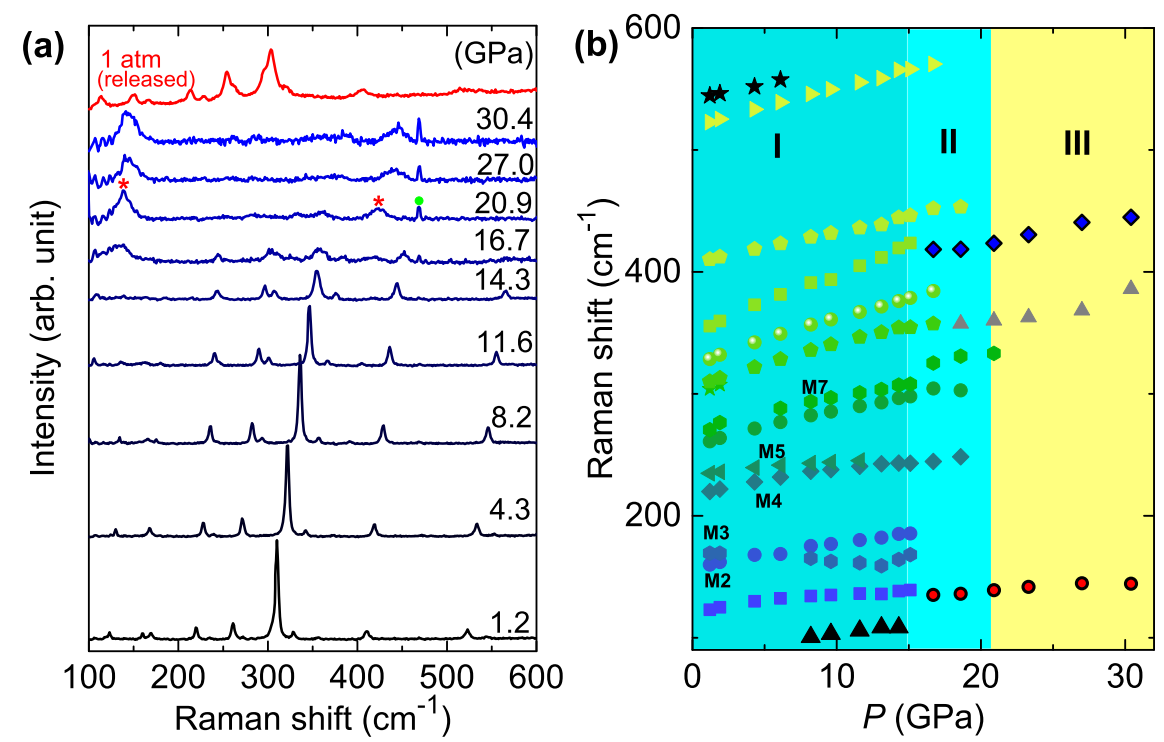

Fig. 5 High-pressure Raman spectroscopy measurements. a Selected Raman spectra of $\mathrm{CrPS}_{4}$ at various pressures. Asterisks mark the strongest Raman modes in the HP phase of $\mathrm{CrPS}_{4}$. The dot represents an unknown Raman mode. $\mathbf{b}$ Pressure dependence of Raman shifts.

resistivity rapidly drops from $\sim 10^{5} \Omega \mathrm{cm}$ at $10 \mathrm{GPa}$ to a less than $\sim 10^{-1} \Omega \mathrm{cm}$ above $\sim 15 \mathrm{GPa}$. Such a huge drop in resistivity indicates that $\mathrm{CrPS}_{4}$ undergoes a pressure-induced insulator-metal transition above $\sim 15 \mathrm{GPa}$, which is further confirmed by the temperature-dependent data shown in Fig. 4 (b). The insulating behavior is recovered upon releasing pressure, which indicates a reversible insulator-metal transition in $\mathrm{CrPS}_{4}$. The corresponding conductivity data (Supplementary Fig. 6) follows a scaling behavior of $\left(\sigma-\sigma_{c}\right) \sim\left(P-P_{c}\right)^{\frac{1}{\gamma}}$ with a critical exponent of $\gamma=3$ and the critical pressure $P_{\mathrm{c}}$ of 13(1) GPa. Similar scaling behavior has been observed in the conductivity of $\mathrm{Cr}$ doped $\mathrm{V}_{2} \mathrm{O}_{3}{ }^{48}$ and $\mathrm{PbCrO}_{3}{ }^{49}$.

The temperature-dependent data below $10 \mathrm{GPa}$ measured from room temperature to $420 \mathrm{~K}$ (inset of Fig. $4(\mathrm{~b})$ ) reveals that the resistivity decreases as the temperature is increased, thus, confirming the insulating behavior of $\mathrm{CrPS}_{4}$ at low pressures. Similarly, resistivity data at $13.6 \mathrm{GPa}$ measured down to $50 \mathrm{~K}$ also shows that the sample possesses semiconducting behavior where the resistivity increases as the temperature is lowered. Further compression leads to the suppression of the semiconducting behavior, and the temperature-dependence data collected above 19 GPa shows a positive slope confirming the fully metallic state of $\mathrm{CrPS}_{4}$ at high pressures, consistent with the optical absorption data. No drop in resistance nor zero resistance at low temperature was observed in the metallic state of $\mathrm{CrPS}_{4}$ thus ruling out the emergence of pressure-induced superconductivity, such as those observed in other van der Waals magnetic insulators such as $\mathrm{FePSe}_{3}{ }^{25}$ and $\mathrm{CrSiTe}_{3}{ }^{29}$.

\section{High-pressure Raman spectrocopy}

High-pressure Raman spectroscopy measurements were then performed to provide insights into the vibrational properties of $\mathrm{CrPS}_{4}$ under pressure. Figure 5(a) shows Raman spectra of $\mathrm{CrPS}_{4}$ measured at various pressures from 1.2 up to $32 \mathrm{GPa}$. The Raman spectrum taken at the lowest pressure of $1.2 \mathrm{GPa}$ consists of 13 active Raman modes, consistent with the Raman spectrum at ambient conditions ${ }^{30,34}$. Upon compression to $10 \mathrm{GPa}$ (region I), most of the Raman modes shift towards higher frequencies (hardens) except the M2 mode at around $170 \mathrm{~cm}^{-1}$, which softens with increasing pressure (Fig. 5(b) and Supplementary Fig. 7). Interestingly, the relative intensity between the M6 and M7 modes $\left(I_{6} / I_{7}\right)$ anomalously increases above $5 \mathrm{GPa}$, whereas the $\mathrm{M} 2$ and $\mathrm{M} 3$
Raman modes seem to undergo a crossover above $4 \mathrm{GPa}$ (Supplementary Fig. 7). Despite these changes, no significant differences were observed in the Raman spectra up to $14 \mathrm{GPa}$.

Between 15 and $20 \mathrm{GPa}$ (region II), we observed the disappearance of several Raman modes observed in region I, as well as the emergence of new Raman modes. The intensities of all Raman peaks are weakened above $15 \mathrm{GPa}$ due to the fact that the sample approaches its fully metallic state. Above $20 \mathrm{GPa}$ (region III), where optical absorption and resistivity data show a metallic behavior, the Raman spectra consist of three Raman modes corresponding to the high-pressure phase of $\mathrm{CrPS}_{4}$. Further compression does not lead to the appearance of new Raman modes and no changes in the Raman spectra are observed up to $30.4 \mathrm{GPa}$. Similar to the electrical transport data, the Raman spectrum returns to its original feature upon decompression to ambient pressure.

Electronic structures of $\mathrm{CrPS}_{4}$ at high pressure

To understand the change in electronic structures observed experimentally, we performed density functional theory (DFT) and DFT + dynamical mean field theory (DMFT) calculations. Owing to the lack of a detailed crystal structure under pressure, we first optimized the structure using the DFT $+U$ method. We note that the additional phonon and Raman calculations we conducted at 0 and $10 \mathrm{GPa}$ to verify the predicted crystal structure under pressure revealed no dynamical instabilities both at 0 and $10 \mathrm{GPa}$ (Supplementary Fig. 8). The calculated Raman spectra are also similar to those measured experimentally (see Supplementary Fig. 8), indicating that our analysis based on the optimized structure from the ab initio calculation (shown below) is reasonable despite the absence of X-ray diffraction data.

Based on the optimized structure from the DFT $+U$ calculations, we performed a paramagnetic (PM) DFT + DMFT calculation because the optical properties under pressure were collected at room temperature (much higher than $T_{\mathrm{N}} \sim 38 \mathrm{~K}$ ). The calculated band structure at $T \sim 298 \mathrm{~K}$ are presented in Fig. 6 . Owing to the strong electron correlation effect, half-filled $\mathrm{Cr}_{2 g}$ orbitals split into lower Hubbard bands and upper Hubbard band, making a Mott gap (Supplementary Fig. 9). $\mathrm{Cr} e_{g}$ bands are located around $2.5 \mathrm{eV}$ while the occupied $S p$ bands dominate the valence band (VB) below $-1 \mathrm{eV}$. $\mathrm{CrPS}_{4}$ at ambient pressure shows a direct-band gap at the $\Gamma$ point. The band gap is estimated to be around $1.5 \mathrm{eV}$, which is governed by the Hubbard bands of $\mathrm{Cr}_{2 g}$ orbitals. Owing 

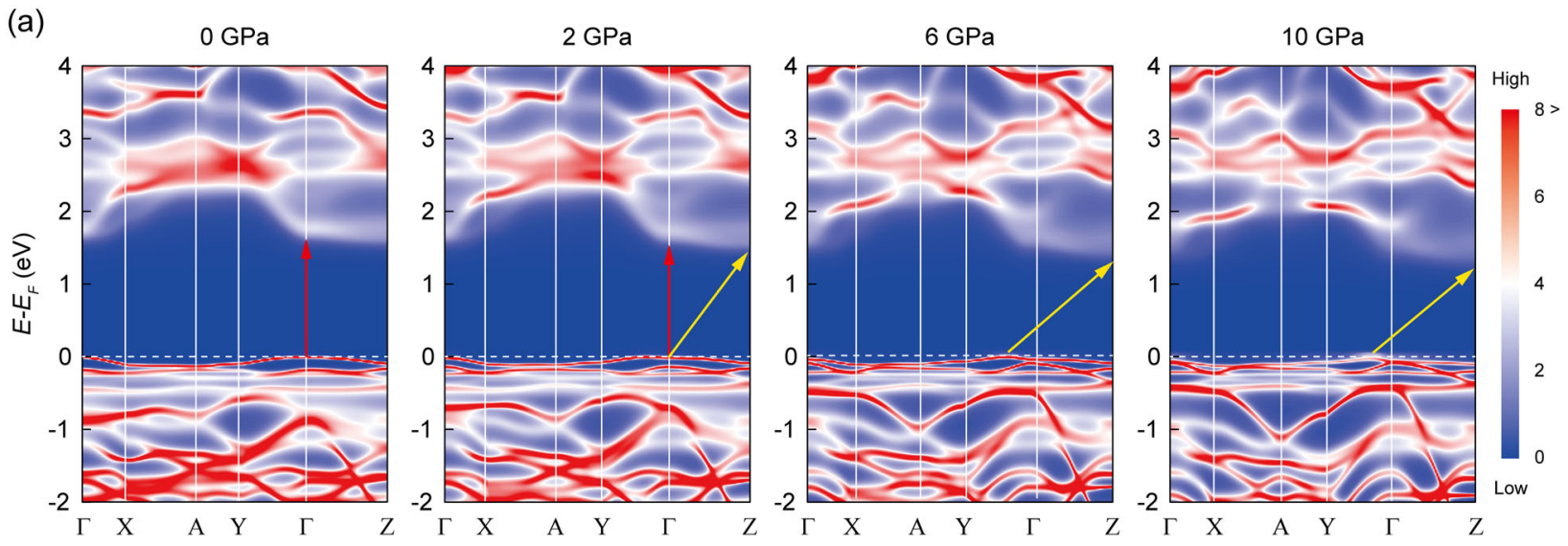

(b)

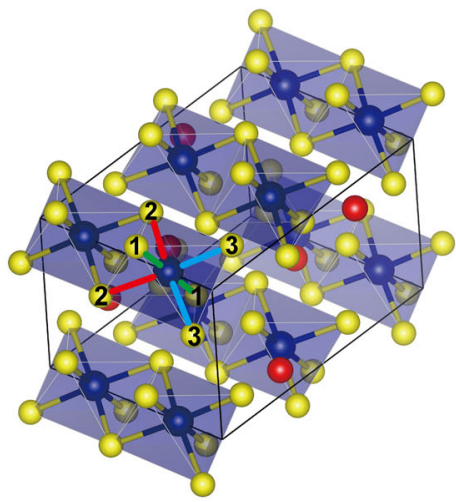

(c)

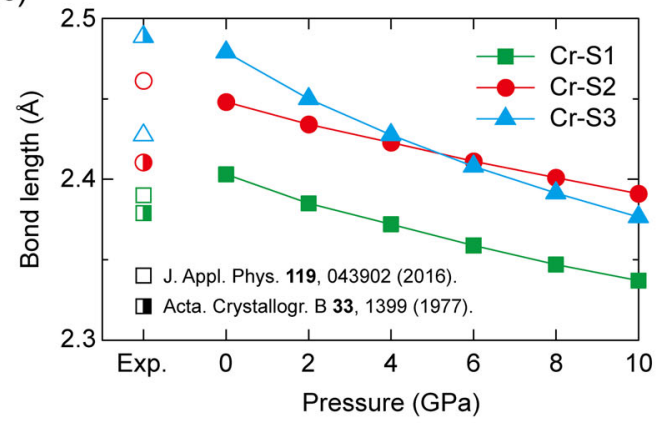

Fig. 6 Theoretical calculations on $\mathrm{CrPS}_{4}$. a Band structures of $\mathrm{CrPS}_{4}$ at $298 \mathrm{~K}$ calculated at various pressures. The $k$-points $\Gamma, X, A, Y, Z$ represent the $(0,0,0),(0.5,0,0),(0.5,0.5,0),(0,0.5,0)$, and $(0,0,0.5)$ points in the conventional Brillouin zone. $\mathbf{b}$ The $\mathrm{CrS}_{6}$ octahedra environment in $\mathrm{CrPS}_{4}$ and $\mathrm{c}$ pressure dependence of the calculated $\mathrm{Cr}-\mathrm{S}$ bond lengths.

to the nearly flat conduction band $(\mathrm{CB})$ along the $\Gamma-Z$ direction, an indirect transition between VB at $\Gamma$ point and $C B$ at $Z$ point cannot be ruled out. The calculated energy gap is consistent with the observation of the PL signal centered at around $1.3 \mathrm{eV}$.

One of the main changes in electronic structure under pressure is the band dispersion in $C B$ along the $\Gamma-Z$ direction. The $Z$ point energy of $C B$ is downshifted compared with the $\Gamma$ point due to the decrease in the interlayer distance as pressure increases. At $2 \mathrm{GPa}$, this shift of the $C B$ slightly changes the nature of the band gap, from a direct to indirect gap between VB at the $\Gamma$ point and $C B$ at the $Z$ point. Above $2 \mathrm{GPa}$, another noticeable change is observed where the second highest VB moves upward (see the $\Gamma$ point). As a result, the VB maximum shifts from $\Gamma$ to between $\Gamma$ and $Y$ points at $6 \mathrm{GPa}$ so that the momentum mismatch of the indirect transition increases. This indicates that the anomalous behaviors of the PL and absoption data of $\mathrm{CrPS}_{4}$ above $3 \mathrm{GPa}$ is related to the direct to indirect band gap crossover (Fig. 2).

To understand the origin of the direct-indirect crossover, we investigated the pressure dependence of the bond length of the $\mathrm{Cr}-\mathrm{S}$ bonds based on the optimized crystal structure obtained from the DFT $+U$ calculations. Owing to the crystal symmetry, there are three types of $\mathrm{Cr}-\mathrm{S}$ bonds in the $\mathrm{CrS}_{6}$ octahedra as shown in Fig. 6(b). The calculated $\mathrm{Cr}-\mathrm{S}$ bond lengths at ambient pressure are well matched with the available experimental results, where the $\mathrm{Cr}-\mathrm{S} 3$ bond is the longest in the octahedra. Upon compression, the $\mathrm{Cr}-\mathrm{S} 3$ bond length decreases much faster than the $\mathrm{Cr}-\mathrm{S} 2$ bond length resulting in a bond length crossover where the $\mathrm{Cr}-\mathrm{S} 2$ bond becomes the longest above $5 \mathrm{GPa}$ (Fig. 6(c)). This bond length crossover agrees well with the VB maximum crossover discussed above. As the bond length difference between $\mathrm{Cr}$-S2 and $\mathrm{Cr}-\mathrm{S} 3$ decreases, the second highest VB originating from the $\mathrm{Cr}_{2 g}$ band moves upward (Supplementary
Fig. 9). Above $5 \mathrm{GPa}$ where the $\mathrm{Cr}-\mathrm{S} 2$ bond becomes longer than the $\mathrm{Cr}-\mathrm{S} 3$ bond, the $\mathrm{VB}$ maximum shifts from $\Gamma$ to between the $\Gamma$ and $Y$ point. This implies that the band gap crossover is driven by the shrinkage and rearrangement of the the $\mathrm{CrS}_{6}$ octahedra. We note that the agreement between the $\mathrm{Cr}-\mathrm{S}$ bond length crossover from our calculations and the $M 2$ and $M 3$ modes crossover observed in the experimental and calculated Raman spectra above 4 GPa (Supplementary Figs. 7 and 9) suggests that the M2 and $\mathrm{M} 3$ modes might be related to the vibration of the $\mathrm{Cr}-\mathrm{S}$ bonds in the $\mathrm{CrS}_{6}$ octahedra.

Having found the occurrence of band gap crossover in $\mathrm{CrPS}_{4}$, we then investigated the possible magnetic transformation under pressure as derived from the DFT $+U(U=2 \mathrm{eV})$ calculations. At ambient pressure, we found that the A-type AFM structure is the most energetically favorable state, consistent with previous DFT studies ${ }^{40}$ and recent neutron diffraction experiments $^{35}$. Our calculations suggest that the A-type AFM structure remains stable and no signs of change in the magnetic state is predicted to occur under pressure up to $20 \mathrm{GPa}$ (see Supplementary Fig. 10). This suggests that the magnetism of $\mathrm{CrPS}_{4}$ is not affected by the band gap crossover. However, further studies using susceptibility measurements under high pressure will clearly be beneficial to investigate the evolution of magnetism of $\mathrm{CrPS}_{4}$ experimentally.

Finally, the nature of the pressure-induced insulator-metal transition in $\mathrm{CrPS}_{4}$ is addressed. As shown above, the Raman spectroscopy experiments suggest a possible structural transition accompanying the insulator-metal transition that occurs above $15 \mathrm{GPa}$. However, whether the structural transformation is an isostructural transition or rather involves the formation of a highpressure phase remains unclear. Based on the optimized structure from DFT $+U$ calculations and assuming that the monoclinic $C / 2 m$ 
space group is stable at high pressure, our DFT + DMFT calculation at $20 \mathrm{GPa}$ shows that $\mathrm{CrPS}_{4}$ remains an insulator with a band gap of $0.7 \mathrm{eV}$. We also calculated the electronic structure at $20 \mathrm{GPa}$ using smaller interaction parameters $(U=8 \mathrm{eV}, J=0.8 \mathrm{eV})$. However, the system is still a Mott insulator with a band gap of $0.4 \mathrm{eV}$. Our DFT + DMFT calculations indicate that the metallization based on the $C / 2 m$ structure occurs at much higher pressure $(P>30 \mathrm{GPa})$ than that observed in the experiment. This implies that the insulator-metal transition in $\mathrm{CrPS}_{4}$ could be accompanied by the formation of a new high-pressure phase as inferred from the Raman spectroscopy data. While the pressure dependence of conductivity follows a mean field exponent (an indication of a Mott transition), it appears that the mechanism behind the insulator-metal transition in $\mathrm{CrPS}_{4}$ cannot be solely attributed to the bandwidth-controlled Mott transition and the formation of high-pressure phase might play an important role in the metallization process. More $\mathrm{X}$-ray diffraction experiments will be carried out in the near future to determine the nature of the structural transition above $15 \mathrm{GPa}$.

The observation of a direct to indirect band gap crossover and insulator-metal transition in the layered antiferromagnetic insulator $\mathrm{CrPS}_{4}$ under pressure has been demonstrated. While the band gap crossover above $3 \mathrm{GPa}$ is due to the shrinkage and rearrangement of the $\mathrm{CrS}_{6}$ octahedra without a change in the crystal symmetry, it appears that the insulator-metal transition could be accompanied by a structural phase transition above $15 \mathrm{GPa}$. These highly tunable properties highlight the strong interplay between the structural, optical, electronic and magnetic properties in $\mathrm{CrPS}_{4}$ and show great potential for device applications based on van der Waals materials.

\section{METHODS}

\section{Sample preparation and characterizations}

A single crystal of $\mathrm{CrPS}_{4}$ was prepared by the chemical vapor transport method. The stoichiometry ratio of $\mathrm{Cr}, \mathrm{P}$, and $\mathrm{S}$ powders were mixed and put into a sealed quartz tube. The sealed tube was then put into a twozone tube furnace and heated up for $24 \mathrm{~h}$. The hot side was $730^{\circ} \mathrm{C}$ while the cold side was about $680^{\circ} \mathrm{C}$, and was maintained for 7 days, then slowly cooled down to room temperature with $100^{\circ} \mathrm{Ch}^{-1}$. X-ray diffraction patterns of the single crystal and pulverized samples were collected using the Rigaku Miniflex diffractometer (Cu-Ka radiation) (Supplementary Notes). The magnetic properties were measured by the Quantum Design magnetic property measurement system (MPMS-XL5) (Supplementary Notes).

\section{High-pressure experiments}

The resistivity data at high pressure were collected in two runs. In the first run, we measured the high-pressure resistivity data with a symmetric diamond anvil cell (DAC) at ambient temperature using a Keithley 6221 current source, 2182A nanovoltmeter, and a 7001 switch system as the current source, voltmeter, and voltage/current switcher, respectively. In the second run, we measured the resistivity of $\mathrm{CrPS}_{4}$ from 300 to $2 \mathrm{~K}$ up to $\sim 31 \mathrm{GPa}$ in a homemade multifunctional measurement system $(1.6-300 \mathrm{~K}$; 0-9 T, Cryomagnetics Inc.) using a DAC made of a Be-Cu alloy. The size of the diamond culet used was $300 \mu \mathrm{m}$ in diameter for all runs. A mixture of epoxy and Cubic boron nitride ( $\mathrm{CBN}$ ) powder was used as a sample chamber and acted as an insulating layer between the electrodes and the stainless steel gasket. The $\mathrm{CrPS}_{4}$ single crystal was cut into a square of approximately $60 \mu \mathrm{m}$ in width and $10 \mu \mathrm{m}$ in thickness. Soft hBN powder was used as a pressure medium. We used the van der Pauw four probe method to measure the electrical resistance using platinum (Pt) foil as electrodes. Several ruby balls were loaded into the sample chamber as pressure calibrants ${ }^{50}$.

The photoluminescence (PL) and Raman measurements were performed in a DAC at room temperature. A small piece of single crystal together with several ruby balls, were placed in a stainless steel gasket with a hole of $120 \mu \mathrm{m}$ diameter. For the measurement of the Raman and PL emission spectra, a Renishaw in Via Raman system with a laser of $532 \mathrm{~nm}$ wavelength was used as an excitation source. High-pressure ultra-visible absorption measurements were performed by a UV-Vis Absorption and Transmission Spectrometer System in a DAC with type-II diamonds up to $25 \mathrm{GPa}$. A mixture of methanol-ethanol (4:1 volume ratio) or silicone oil was used as a pressure transmitting medium.

\section{Theoretical calculations}

To study the correlated electronic structure of $\mathrm{CrPS}_{4}$, we employed the embedded DMFT combined with DFT (DFT + DMFT) as implemented in the WIEN2k + eDMFT package ${ }^{51}$. The WIEN2 $\mathrm{k}$ calculations were performed using the PBE-GGA functional ${ }^{52}$ with $1000 k$-points. The correlation effect of $\mathrm{Cr} 3 d$ orbitals was treated by a DMFT loop on the top of an effective one-electron Hamiltonian generated from the WIEN2k calculation. A hybridization energy window from -10 to $10 \mathrm{eV}$ with respect to the Fermi level was chosen, and the Coulomb interaction of $U=10 \mathrm{eV}$ and Hund's coupling of $J=1 \mathrm{eV}$ were used for the $\mathrm{Cr} d$ orbitals. The local impurity problem was solved by using continuous time quantum Monte Carlo $(C T Q M C)^{53}$. For the structural optimization at different pressure, we used the Vienna ab initio package (VASP) ${ }^{54}$, where the plane-wave cut off was set to $500 \mathrm{eV}$. The DFT-D3 functional was used to describe the van der Waals (vdW) interaction ${ }^{55}$.

\section{DATA AVAILABILITY}

All relevant data are available from the authors upon reasonable request.

Received: 15 April 2020; Accepted: 28 July 2020;

Published online: 18 August 2020

\section{REFERENCES}

1. Novoselov, K. S. et al. Two-dimensional atomic crystals. Proc. Natl Acad. Sci. USA 102, 10451-10453 (2005).

2. Park, J.-G. Opportunities and challenges of 2D magnetic van der Waals materials: magnetic graphene? J. Phys. Condens. Matter 28, 301001 (2016).

3. Samarth, N. Magnetism in flatland. Nature 546, 216-217 (2017).

4. Burch, K. S., Mandrus, D. \& Park, J.-G. Magnetism in two-dimensional van der Waals materials. Nature 563, 47-52 (2018).

5. Lee, J. U. et al. Ising-type magnetic ordering in atomically thin $\mathrm{FePS}_{3}$. Nano Lett. 16, 7433-7438 (2016).

6. Gong, C. et al. Discovery of intrinsic ferromagnetism in two-dimensional van der Waals crystals. Nature 546, 265-269 (2017).

7. Huang, B. et al. Layer-dependent ferromagnetism in a van der Waals crystal down to the monolayer limit. Nature 546, 270-273 (2017).

8. Fei, Z. et al. Two-dimensional itinerant ferromagnetism in atomically thin $\mathrm{Fe}_{3} \mathrm{GeTe}_{2}$. Nat. Mater. 17, 778-782 (2018).

9. Deng, Y. et al. Gate-tunable room-temperature ferromagnetism in twodimensional $\mathrm{Fe}_{3} \mathrm{GeTe}_{2}$. Nature 563, 94-99 (2018).

10. Kim, K. et al. Large anomalous Hall current induced by topological nodal lines in a ferromagnetic van der waals semimetal. Nat. Mater. 17, 794-799 (2018).

11. Seo, J. et al. Nearly room temperature ferromagnetism in a magnetic-metal-rich van der Waals metal. Sci. Adv. 6, eaay8912 (2020).

12. Léaustic, A. et al. High- $T_{c}$ magnets in a series of substituted pyridinium $\mathrm{FePS}_{3}$ layered intercalates. Chem. Mater. 8, 1954-1961 (1996).

13. Evans, J. S. O. et al. Origins of the spontaneous magnetization in $\mathrm{MnPS}_{3}$ intercalates: a magnetic susceptibility and powder neutron diffraction study. Adv. Mater. 7, 735-739 (1995).

14. Jiang, $\mathrm{S}$. et al. Controlling magnetism in $2 \mathrm{D} \mathrm{Crl}_{3}$ by electrostatic doping. Nat. Nanotech. 13, 549-553 (2018).

15. Wang, Z. et al. Electric-field control of magnetism in a few-layered van der Waals ferromagnetic semiconductor. Nat. Nanotech. 13, 554-559 (2018).

16. Ciarrocchi, A., Avsar, A., Ovchinnikov, D. \& Kis, A. Thickness-modulated metal-tosemiconductor transformation in a transition metal dichalcogenide. Nat. Commun. 9, 919 (2018).

17. Avsar, A. et al. Defect induced, layer-modulated magnetism in ultrathin metallic $\mathrm{PtSe}_{2}$. Nat. Nanotechnol. 14, 674-678 (2019).

18. Wang, Y. et al. Pressure-driven cooperative spin-crossover, large-volume collapse, and semiconductor-to-metal transition in Manganese(II) honeycomb lattices. J. Am. Chem. Soc. 138, 15751-15757 (2016).

19. Lin, Z. et al. Pressure-induced spin reorientation transition in layered ferromagnetic insulator $\mathrm{Cr}_{2} \mathrm{Ge}_{2} \mathrm{Te}_{6}$. Phys. Rev. Mater. 2, 051004 (2018).

20. Haines, C. R. S. et al. Pressure-induced electronic and structural phase evolution in the van der Waals compound FePS 3 . Phys. Rev. Lett. 121, 266801 (2018). 
21. $\mathrm{Li}, \mathrm{T}$. et al. Pressure-controlled interlayer magnetism in atomically thin $\mathrm{Crl}_{3}$. Nat. Mater. 18, 1303-1308 (2019).

22. Song, T. et al. Switching 2D magnetic states via pressure tuning of layer stacking. Nat. Mater. 18, 1298-1302 (2019).

23. Mondal, S. et al. Effect of hydrostatic pressure on ferromagnetism in twodimensional $\mathrm{Crl}_{3}$. Phys. Rev. B 99, 180407 (2019).

24. Wang, $X$. et al. Pressure-induced modification of the anomalous Hall effect in layered $\mathrm{Fe}_{3} \mathrm{GeTe}_{2}$. Phys. Rev. B 100, 014407 (2019).

25. Wang, Y. et al. Emergent superconductivity in an iron-based honeycomb lattice initiated by pressure-driven spin-crossover. Nat. Commun. 9, 1914 (2018).

26. Coak, M. J. et al. Isostructural Mott transition in $2 \mathrm{D}$ honeycomb antiferromagnet $\mathrm{V}_{0.9} \mathrm{PS}_{3}$. NPJ Quant. Mater. 4, 38 (2019).

27. Coak, M. J. et al. Tuning dimensionality in van-der-Waals antiferromagnetic Mott insulators $\mathrm{TMPS}_{3}$. J. Phys.: Condens. Matter 32, 124003 (2020).

28. Ahmad, A. S. et al. Pressure-driven switching of magnetism in layered $\mathrm{CrCl}_{3}$. Preprint at https://arxiv.org/abs/1911.03846 (2019).

29. Cai, W. et al. Pressure-induced superconductivity and structural transition in ferromagnetic $\mathrm{Cr}_{2} \mathrm{Si}_{2} \mathrm{Te}_{6}$. Preprint at https://arxiv.org/abs/1912.05166 (2019).

30. Lee, J. et al. Structural and optical properties of single- and few-layer magnetic semiconductor $\mathrm{CrPS}_{4}$. ACS Nano 11, 10935-10944 (2017).

31. Lee, M. J. et al. Synaptic devices based on two-dimensional layered single-crystal Chromium Thiophosphate $\left(\mathrm{CrPS}_{4}\right)$. NPG Asia Mater. 10, 23-30 (2018).

32. Kim, S. et al. Crossover between photochemical and photothermal oxidations of atomically thin magnetic semiconductor $\mathrm{CrPS}_{4}$. Nano Lett. 19, 4043-4051 (2019).

33. $\mathrm{Wu}, \mathrm{H}$. \& Chen, H. Probing the properties of lattice vibrations and surface electronic states in magnetic semiconductor $\mathrm{CrPS}_{4}$. RSC Adv. 9, 30655-30658 (2019).

34. Gu, P. et al. Photoluminescent quantum interference in a van der Waals magnet preserved by symmetry breaking. ACS Nano 14, 1003-1010 (2020).

35. Peng, Y. et al. Magnetic structure and metamagnetic transitions in the van der Waals antiferromagnet $\mathrm{CrPS}_{4}$. Adv. Mater. https://onlinelibrary.wiley.com/doi/abs/ 10.1002/adma.202001200 (2020).

36. Diehl, R. \& Carpentier, D. The crystal structure of chromium thiophosphate, $\mathrm{CrPS}_{4}$. Acta Crystallogr. Sect. B 33, 1399-1404 (1977).

37. Louisy, A., Ouvrard, G., Schleich, D. M. \& Brec, R. Physical properties and lithium intercalates of $\mathrm{CrPS}_{4}$. Solid State Commun. 28, 61-66 (1978).

38. Pei, Q. L. et al. Spin dynamics, electronic, and thermal transport properties of twodimensional $\mathrm{CrPS}_{4}$ single crystal. J. Appl. Phys. 119, 043902 (2016).

39. Ohno, Y., Mineo, A. \& Matsubara, I. I. Reflection electron-energy-loss spectroscopy, $\mathrm{X}$-ray-absorption spectroscopy, and X-ray photoelectron spectroscopy studies of a new type of layer compound $\mathrm{CrPS}_{4}$. Phys. Rev. B 40, 10262-10272 (1989).

40. Zhuang, H. L. \& Zhou, J. Density functional theory study of bulk and single-layer magnetic semiconductor $\mathrm{CrPS}_{4}$. Phys. Rev. B 94, 195307 (2016).

41. Wood, D. L., Ferguson, J., Knox, K. \& Dillon, J. F. Crystalfield spectra of $d^{3,7}$ ions. iii. spectrum of $\mathrm{Cr}^{3+}$ in various octahedral crystal fields. J. Chem. Phys. 39, 890-898 (1963).

42. Nosenzo, L., Samoggia, G. \& Pollini, I. Effect of magnetic ordering on the optical properties of transition-metal halides: $\mathrm{NiCl}_{2}, \mathrm{NiBr}_{2}, \mathrm{CrCl}_{3}$, and $\mathrm{CrBr}_{3}$. Phys. Rev. $B$ 29, 3607-3616 (1984).

43. Seyler, K. L. et al. Ligand-field helical luminescence in a $2 D$ ferromagnetic insulator. Nat. Phys. 14, 277-281 (2017).

44. Henderson, B. \& Imbusch, G. F. Optical Spectroscopy of Inorganic Solids. (Clarendon Press, Oxford, 1989).

45. Lee, C.-J. et al. Observation of pressure-induced direct-to-indirect band gap transition in InP nanocrystals. J. Chem. Phys. 113, 2016 (2000).

46. Manjón, F. J. et al. Band structure of indium selenide investigated by intrinsic photoluminescence under high pressure. Phys. Rev. B 70, 125201 (2004).

47. $\mathrm{Fu}$, L. et al. K- $\lambda$ crossover transition in the conduction band of monolayer $\mathrm{MoS}_{2}$ under hydrostatic pressure. Sci. Adv. 3, e1700162 (2017).

48. Limelette, P. et al. Universality and critical behavior at the Mott transition. Science 302, 89-92 (2003).

49. Wang, S. et al. Unusual Mott transition in multiferroic $\mathrm{PbCrO}_{3}$. Proc. Natl Acad. Sci. USA 112, 15320-15325 (2015).

50. Mao, H. K., Xu, J. \& Bell, P. M. Calibration of the ruby pressure gauge to $800 \mathrm{kbar}$ under quasihydrostatic conditions. J. Geophys. Res. 91, 4673-4676 (1986).

51. Haule, K., Yee, C.-H. \& Kim, K. Dynamical mean-field theory within the fullpotential methods: Electronic structure of Celrln ${ }_{5}, C_{e C o l n}$, and CeRhln ${ }_{5}$. Phys. Rev. B 81, 195107 (2010).
52. Perdew, J. P., Burke, K. \& Ernzerhof, M. Generalized gradient approximation made simple. Phys. Rev. Lett. 78, 1396 (1997).

53. Haule, K. Quantum Monte Carlo impurity solver for cluster dynamical mean-field theory and electronic structure calculations with adjustable cluster base. Phys. Rev. B 75, 155113 (2007).

54. Kresse, G. \& Furthmüller, J. Efficient iterative schemes for ab initio total-energy calculations using a plane-wave basis set. Phys. Rev. B 54, 11169-11186 (1996).

55. Grimme, S., Antony, J., Ehrlich, S. \& Krieg, H. A consistent and accurate ab initio parametrization of density functional dispersion correction (DFT-D) for the 94 elements H-Pu. J. Chem. Phys. 132, 154104 (2010).

\section{ACKNOWLEDGEMENTS}

We acknowledge support from the National Natural Science Foundation of China (NSFC) under grant numbers U1530402, 11774015, and 11811530001. This work was supported by the U.S. DOE-BES, Division of Materials Science and Engineering, under Contract No. DE-SC0012704 (BNL materials synthesis). H.L.D. acknowledges the support from the Opening Project of State Key Laboratory of High Performance Ceramics and Superfine Microstructure (Project No. SKL201802SIC.). We thank Saqib Rahman and Nana Li for their help with resistivity measurements, and Yanping Yang for her assistance with EDS measurements. We thank Freyja O'Toole for her assistance with the language editing.

\section{AUTHOR CONTRIBUTIONS}

R.A.S., B.G.Y., and B.C. designed and conceived the project. Q.D. and C.P. synthesized the sample and carried out basic characterizations. R.A.S., J.J.F., and H.L.D. carried out resistivity measurements. Z.Y. performed the UV-Vis optical absorption experiments. R.A.S. and M.Z.Y. performed photoluminescence and Raman spectroscopy experiments. B.G.J., J.H.S., and D.Y.K. performed theoretical calculations. R.A.S., B.G.J, and J.J.F. analyzed the data and wrote the paper with the contributions from other co-authors.

\section{COMPETING INTERESTS}

The authors declare no competing interests.

\section{ADDITIONAL INFORMATION}

Supplementary information is available for this paper at https://doi.org/10.1038/ s41535-020-00261-x.

Correspondence and requests for materials should be addressed to R.A.S. or B.C.

Reprints and permission information is available at http://www.nature.com/ reprints

Publisher's note Springer Nature remains neutral with regard to jurisdictional claims in published maps and institutional affiliations.

(i) Open Access This article is licensed under a Creative Commons adaptation, distribution and reproduction in any medium or format, as long as you give appropriate credit to the original author(s) and the source, provide a link to the Creative Commons license, and indicate if changes were made. The images or other third party material in this article are included in the article's Creative Commons license, unless indicated otherwise in a credit line to the material. If material is not included in the article's Creative Commons license and your intended use is not permitted by statutory regulation or exceeds the permitted use, you will need to obtain permission directly from the copyright holder. To view a copy of this license, visit http://creativecommons. org/licenses/by/4.0/.

(c) The Author(s) 2020 